\title{
Carpal Tunnel Release Outcomes in Diabetic Versus Non-Diabetic Patients
}

\author{
Kamran Mozaffarian ${ }^{1}$; Mohammad Owjimehr ${ }^{1}$; Behdad Eskandari Sani ${ }^{1}$; Farzam Mokarami \\ ${ }^{2, *} ;$ Reza Sharifzadeh $^{2}$ \\ ${ }_{1}^{1}$ Bone and Joint Disease Research Center, Shiraz University of Medical Sciences, Shiraz, IR Iran \\ 2 Department of Orthopedic Surgery, Imam Reza Hospital, AJA University of Medical Sciences, Tehran, IR Iran \\ ${ }^{*}$ Corresponding author: Farzam Mokarami, Department of Orthopedic Surgery, Imam Reza Hospital, AJA University of Medical Sciences, Tehran, IR Iran. Tel: + 98-2188028931, \\ E-mail:farzam_m2001@yahoo.com
}

Received: December 22, 2014; Revised: January 15, 2015; Accepted: February 23, 2015

Background:Carpal tunnel syndrome(CTS) is the most common compressive neuropathy. Diabetes mellitus is the most common disease that predisposes the patients to CTS. Diabetic neuropathy is a progressive disease and diabetics nerve is more susceptible to compression at known sites of anatomic constrains such as in the carpal tunnel; clinical results of carpal tunnel release has been questioned not to be as good as non-diabetic patients.

Objectives: This study was carried out to compare the clinical and electrodiagnostic outcomes of diabetic and non-diabetic carpal tunnel release (CTR) surgery.

Patients and Methods: Twenty diabetic hands (14 patients) and 18 non-diabetic hands (14 patients) that underwent CTR between Octobers 2009 - 2012 were evaluated. They were operated by one hand surgeon and were evaluated at least six months after the operation. Clinical symptoms as numbness, pain, paresthesia and nocturnal symptoms were evaluated. Electrodiagnostic results were evaluated pre- and post-operatively.

Results: After surgical release, both groups showed significant improvements in clinical results, as $81.6 \%$ of patients had excellent and good outcomes, $18.4 \%$ had fair outcomes, and no one had poor outcome. Except for median sensory nerve conduction velocity (NCV) changes that had significant differences between diabetic and non-diabetic groups, other parameters had no significant differences between both groups. Furthermore, significant reverse correlation was found between the duration of CTS and the outcome.

Conclusions: Clinical and electrodiagnostic results after CTR are approximately the same in patients with diabetes and without it. Diabetics with CTS as well as non-diabetic patients have satisfactory results after CTR surgery. Furthermore, the duration of CTS has a significant influence on the result of CTR; the shorter duration of diabetes, the better the result of CTR surgery.

Keywords: Carpal Tunnel Syndrome; Diabetes Mellitus; Carpal Tunnel Release

\section{Background}

Carpal tunnel syndrome (CTS) is the most common compressive neuropathy $(1,2)$. It has several risk factors, the most common of which is diabetes mellitus (DM) (1, 3). In diabetic patients, median nerve neuropathy may have two common causes which are diabetic neuropathy and CTS, or both of them simultaneously (3). Due to diabetic neuropathy, structural nerve changes such as segmental and paranodal demyelination cause a decrease in nerve conduction velocity (NCV); also, loss of axons in nerve trunk may decrease the nerve action potential $(4,5)$. On the other hand, CTS is caused due to compression of median nerve in carpal tunnel and is a variant of compressive neuropathy; it causes elevation in carpal tunnel pressure more than 20 - $30 \mathrm{mmHg}$ which can lead to reduction of epineurial venule blood flow and inhibition of both ante grade and retrograde axonal transport. These changes are transitory and therefore reversible in short term, but prolonged pressure can result in lasting damages. There is some controversy about the outcome of carpal tunnel release between diabetic and non-diabetic patients. Some studies have shown less favorable results in diabetic patients $(6,7)$, but other studies have shown same outcomes between the two groups $(8,9)$. Carpal tunnel release (CTR) has different techniques including open and endoscopic (10-13). Open technique can be performed with one inch skin incision $(2-3 \mathrm{~cm})$ ulnar and paralleling to the thenar crease which is called mini open technique; this (14-18) is the CTR method in our study.

\section{Objectives}

With regard to the high incidence of DM in the population and the high prevalence of CTS in diabetic and 
non-diabetic patients and also because of the differences between the results of previous studies, in this study, we aim to compare the clinical and electrodiagnostic outcomes between diabetic and non-diabetic patients.

\section{Patients and Methods}

The study was approved by the ethics board of our institution. This was a retrospective study on patients undergone CTR by one experienced hand surgeon. We called up 30 consecutive patients with the diagnosis of CTS who underwent CTR with mini open technique between Octobers 2009-2012. Among these 30 patients, 15 were diabetic and 15 were non-diabetic. The diagnosis of diabetes in diabetic patients was approved by fasting blood sugar above $126 \mathrm{mg} / \mathrm{dL}$ and two hours postprandial above 200 $\mathrm{mg} / \mathrm{dL}$ (19). The diagnosis of CTS was approved by clinical and electrophysiological findings.

Treatment had already been started by an endocrinologist before the surgery. At least, five years had been passed since the commencement of DM diagnosis. For more assurance, we measured blood sugar in non-diabetic patients too. Only patients with moderate or severe CTS who had not responded to conservative measures were enrolled in this study. Patients with one of the following factors were excluded from the study:

1. Polyneuropathy, cervical radiculopathy or other compressive neuropathies which were proved by electrodiagnostic study.

2. Diabetic patients who underwent treatment by insulin, because of insulin ability to increase neurotrophic factors.

3. Patients who were operated by other surgical methods rather than mini open incision.

4. History of rheumatoid arthritis, thyroid or renal disease.

5. Pregnant women.

6. History of fracture or previous surgery on the affected side.

7. Long-term exposure to vibrating devices.

8. Patients with thenar atrophy

\subsection{Clinical Study}

At final follow up of all the patients was evaluated both clinically and electrodiagnostically. Four important factors were evaluated clinically, including numbness, pain, paresthesia and nocturnal symptoms (3). The results of clinical evaluation were graded as follows $(8,9)$ :

1. Excellent: when all symptoms were subsided postoperatively.

2. Good: when all the symptoms relieved except for numbness.

3. Fair: when patient had pain and/or paresthesia during heavy activities or when nocturnal symptoms persisted but milder than preoperative period.

4. Poor: when symptoms were unchanged or minimally improved postoperatively.

\subsection{Electrodiagnostic Study}

Electrodiagnostic test was performed for all the patients and compared with preoperative electrodiagnostic results. We evaluated median nerve motor distal latency (MDL) and sensory distal latency (SDL) and nerve conductive velocity (NCV) in both diabetic and non-diabetics pre-and post-operatively $(5,12)$. All the electrodiagnostic tests were performed before and after the surgery by the same physiatrist.

\subsection{Statistical Analysis}

We preformed the analysis using the Statistical Package for the Social Sciences (SPSS) software version 15. We used chi-squared and Fisher's exact tests to compare the categorical data. Data were compared by t-test and paired $t$ test. $\mathrm{P}<0.05$ was considered statistically significant.

\section{Results}

In each group, one patient refused the electrodiagnostic study; so, 14 diabetic (20 hands) and 14 non-diabetic patients (18 hands) were evaluated. All the diabetic patients were female and in the non-diabetic group only one patient was male. The age range was 30 - 65 years; the minimum follow up was six months. The results of clinical evaluation in diabetic and non-diabetic patients are summarized in Tables 1 - 4. In none of the diabetic or non-diabetic patients pain and paresthesia and nocturnal symptoms persisted postoperatively with the severity as same as the preoperative period. There was no significant difference between the diabetic and non-diabetic groups in clinical improvements after CTR $(\mathrm{P}=0.73)$. Consequently, nocturnal symptom was the most improved complaint and numbness was the most resistant symptom to treatment. The duration of CTS symptoms of before operation was 2 - 14 years (mean: $4.5 \pm 3.8$ ) in diabetic and 2 - 10 years (mean: $4 \pm 3.2$ ) in non-diabetic patients .We the categorized preoperative duration of CTS into three groups:

1. Group DI: $\leq$ three years

2. Group DII: three years $<$ duration $>10$ years

3. Group DIII: $\geq 10$ years

Table 1. Improvement of Numbness in Diabetic and Non-Diabetic Patients ${ }^{\text {a }}$

\begin{tabular}{|c|c|c|c|}
\hline \multirow[t]{2}{*}{ Patients } & \multicolumn{3}{|c|}{ Numbness ${ }^{\mathrm{b}}$} \\
\hline & 1 & 2 & 3 \\
\hline Diabetic & 40 & & 60 \\
\hline Non-diabetic & 33.3 & & 66.7 \\
\hline Total & 36.8 & & 63.2 \\
\hline
\end{tabular}


Table 2. Improvement of Pain in Diabetic and Non-Diabetic Patients ${ }^{\mathrm{a}}$

\begin{tabular}{lcc}
\hline Patients & \multicolumn{3}{c}{ Pain ${ }^{\mathrm{b}}$} \\
\cline { 2 - 3 } & $\mathbf{2}$ & $\mathbf{3}$ \\
\hline Diabetic & 15 & 85 \\
Non-diabetic & 5.6 & 94.4 \\
Total & 10.5 & 89.5 \\
\hline $\begin{array}{l}\text { a Data are presented as \%. } \\
\text { b }{ }_{1} \text {, no improvement or minimal improvement; 2, there is symptoms } \\
\text { only with heavy activities; 3, competent improvement. }\end{array}$
\end{tabular}

Table 3. Improvement of Paresthesia in Diabetic and NonDiabetic Patients ${ }^{\mathrm{a}}$

\begin{tabular}{lcc}
\hline Patients & $\mathbf{3}$ & Paresthesia $^{\mathrm{b}}$ \\
\cline { 2 - 3 } & $\mathbf{2}$ & $\mathbf{3}$ \\
\hline Diabetic & 20 & 80 \\
Non-diabetic & 16.7 & 83.3 \\
Total & 18.4 & 81.6 \\
\hline $\begin{array}{l}\text { a Data are presented as \%. } \\
\text { b 1, no improvement or minimal improvement; 2, there is symptoms } \\
\text { only with heavy activities; 3, competent improvement. }\end{array}$
\end{tabular}

Table 4. Improvement of Nocturnal Symptom in Diabetic and Non-Diabetic Patients a

\begin{tabular}{lccc}
\hline Patients & \multicolumn{3}{c}{ Nocturnal Symptom $^{\mathrm{b}}$} \\
\cline { 2 - 4 } & $\mathbf{1}$ & $\mathbf{2}$ & $\mathbf{3}$ \\
\hline Diabetic & 5 & 95 \\
Non-diabetic & 5.6 & 94.4 \\
Total & 5.3 & 94.7 \\
\hline
\end{tabular}

${ }^{\mathrm{a}}$ Data are presented as \%.

b 1 , no improvement or minimal improvement; 2 , there is symptoms only with heavy activities; 3 , competent improvement.

Final clinical outcomes in DI, DII, and DIII groups are shown in Figures 1 and 2. In diabetic and non-diabetic groups, there was a reverse correlation between the duration of CTS and the outcome in both diabetic and nondiabetic patients. The clinical outcome worsens as the duration of symptoms increase. In this regard, the difference between DI and DII groups was statistically significant $(\mathrm{P}=0.007)$ as well as the difference between DII and DIII groups $(\mathrm{P}=0.00)$. When the duration of symptoms was more than 10 years before the operation, all the nondiabetics and $60 \%$ of the diabetics had fair results. Among 38 hands that underwent CTR, 27 were dominant and 11 were nondominant. There was no significant correlation between the dominancy of hand and the outcome of surgery $(P=0.237)$.

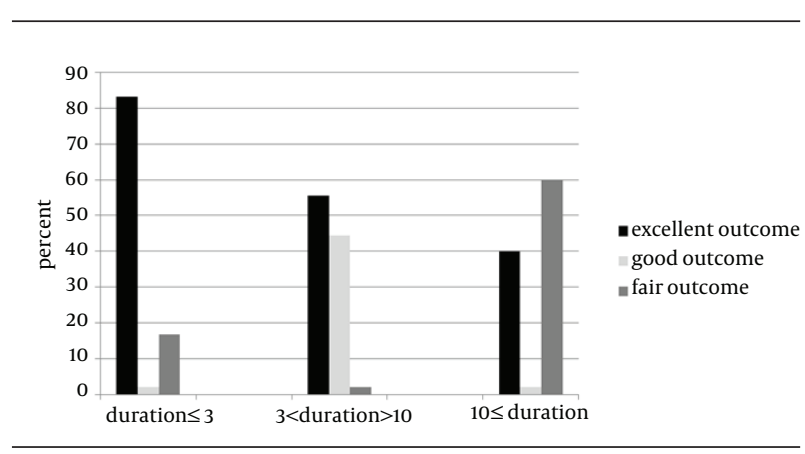

Figure 1. Comparison of Clinical Outcomes in Diabetic Patients Based on Duration of Symptoms Preoperatively

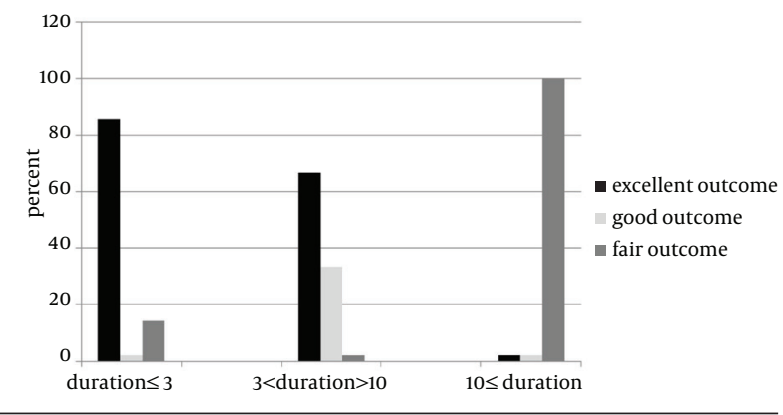

Figure 2. Comparison of Clinical Outcomes in Non-Diabetic Patients Based on Duration of Symptoms Preoperatively

\subsection{Assessment of Electrodiagnostic Results}

Electrodiagnostic study including MDL, SDL and NCV was performed postoperatively and compared with the preoperative results. Both groups showed decrease in MDL postoperatively. The overall MDL decreased from 6.6 \pm 1.4 to $4.5 \pm 0.7$, which was statistically significant $(\mathrm{P}=$ 0.00 ) (Figure 3). The difference between pre- and postoperative MDL was $1.9 \pm 1.4$ in diabetic and $2.2 \pm 1.1$ in non-diabetic patients. Although this improvement in MDL was more in non-diabetic patients than the diabetic group, the difference between non-diabetics and diabetic patients was not statistically significant $(P=0.532)$.

Both groups showed decrease in median nerve SDL postoperatively. The overall SDL decreased from $6.3 \pm 2.3$ to $4.5 \pm 1.8$, which was statistically significant $(\mathrm{P}=0.00)$ (Figure 4). The difference between pre- and postoperative SDL was $2 \pm 1.7$ in diabetic patients and $2 \pm 2$ in non-diabetic ones. Although this change was again more in nondiabetic patients, there was no statistical significance ( $P$ $=0.991$ ). Both groups showed increase in median nerve NCV postoperatively. The overall NCV increased from 19.7 \pm 8.5 to $31 \pm 10.2$, which was statistically significant $(\mathrm{P}=$ 0.00 ) (Figure 5). The difference between pre- and postoperative NCV was $7.7 \pm 5.5$ in diabetic patients and $15 \pm 6.8$ in the non-diabetic group, which showed that improvement in NCV after the operation was much more in nondiabetic patients than the diabetic group and this was statistically significant $(\mathrm{P}=0.008)$. 


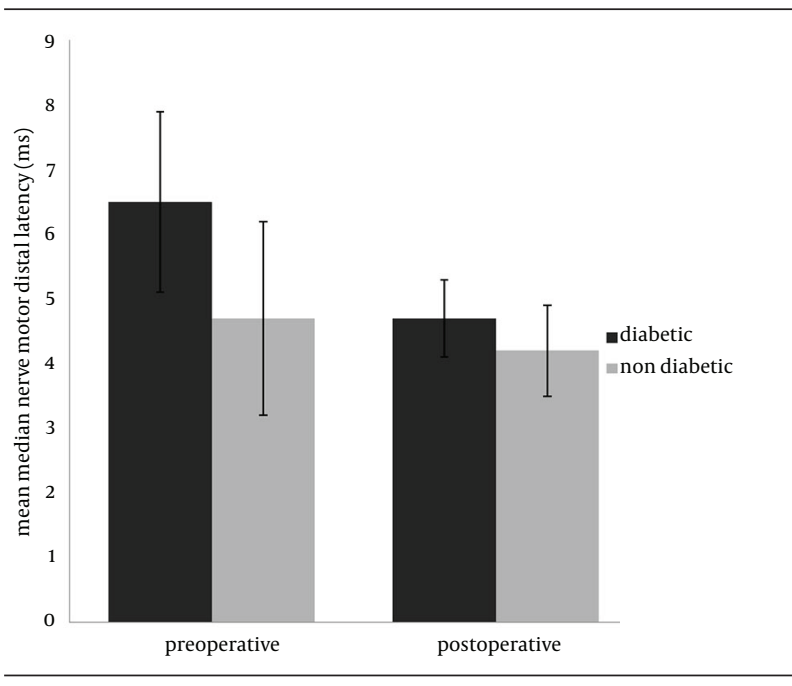

Figure 3. Comparison of Mean Median Nerve Motor Distal Latency in Diabetic and Non-Diabetic Groups Pre- and Postoperatively

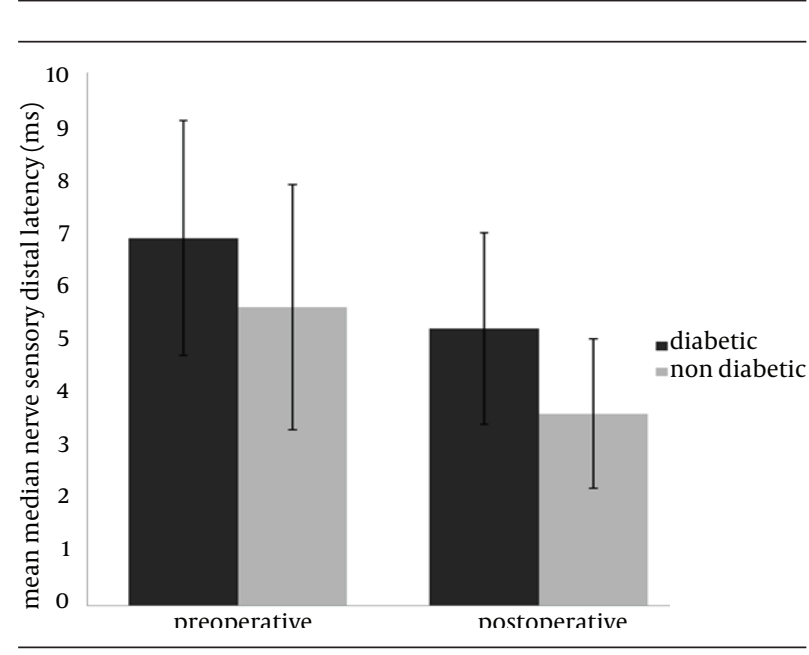

Figure 4. Comparison of Mean Median Nerve Sensory Distal Latency in Diabetic and Non-Diabetic Groups Pre- and Postoperatively

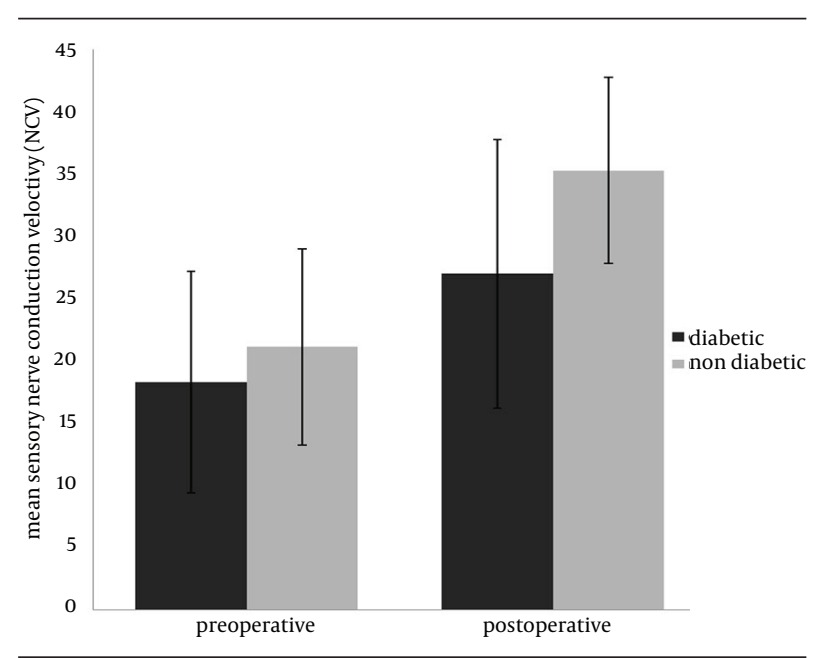

Figure 5. Comparison of Mean Nerve Conduction Velocity in Diabetic and Non-Diabetic Groups Pre- and Postoperatively

\section{Discussion}

CTS has always been debilitating for patients affected by that and moreover in patients with an underlying neuropathic disorder such as DM $(1,3)$, whether CTR can add as much benefit to the diabetic patients as non-diabetic patients has always been questioned. Although some studies have shown less favorable results in diabetic patients after CTR $(11,12)$, Thomsen et al. (9) and Wong et al. (15) presented the same comparable outcome between diabetic and non-diabetic patients. Of different aspects of motor and sensory involvement in CTS, a mindful CTR procedure can resolve most of the patients' complaints (9). The current study evaluated two groups of diabetic and non-diabetic patients after CTR. As previous studies, we attained significant improvements in clinical results (pain, paresthesia and numbness) in both diabetic and non-diabetic patients after operation $(4,9,20)$, as excellent and good outcome was observed in $80 \%$ of diabetic and $83.4 \%$ of non-diabetic patients, whereas there was no poor outcome in both groups and there was fair outcome in $20 \%$ of diabetic and $16.7 \%$ of non-diabetic patients. There was no significant difference between the clinical results of diabetic and non-diabetic patients after the surgery $(\mathrm{P}=0.73)$. This study showed minimal or no improvement in numbness of fingers postoperatively in $40 \%$ of diabetic and $33.3 \%$ of non-diabetic patients, which was almost above average in contrast with $20 \%$ failure rate in other studies $(4,8,11,13)$, which can be due to the disease severity mismatch of our patients and the surgical technique used in other studies. Night (bed time) symptoms and sleep disturbances are the symptoms that mostly responded to surgical release in this study, as $94.7 \%$ of patients had no nocturnal symptoms after the operation and this was the same as what Thomsen and his colleagues reported previously (9). MDL and SDL as well as sensory NCV showed significant improvements after the operation, either in diabetic or in non-diabetic patients, as in other studies $(4,11)$. The level of improvement in MDL and SDL was the same in diabetic and non-diabetic patients, but NCV showed significantly more improvement in non-diabetic patients $(P=0.008)$. This can be explained by the ill-conducting nature of a diabetic neuron. Cold intolerance is another issue of diabetic patients in the study of Thomsen et al. (4) which did not respond well in diabetic patients, though we could not evaluate it in our survey.

On the other hand, although electrodiagnostic study showed improvement of all the parameters postoperatively in all patients in this study, they did not reach the normal level (contrary to symptoms); so, they can neither be used as a predictive tool for successful result of a surgery, nor a correlation between clinical outcome and postoperative improvement of electrodiagnostic parameters existed.

In our research, we found that the duration of CTS before the surgery significantly influenced the outcome 
of CTR in both diabetic and non-diabetic patients; if the symptoms of CTS persist for three years or less, the best outcome can be achieved through CTR and no favorable outcome would be expected if it affected the patient more than 10 years.

One of the limitations of this study was its retrospective nature, so we had no grip power and pinch power before the surgery, and therefore we could not use more popular and reliable scores such as the DASH score. That is why patients with thenar region atrophy were excluded from the evaluation, so we could conclude more reliable results; moreover, larger study population can increase the reproducibility of the results.

In conclusion, this study showed that although surgery caused more improvement in NCV in non-diabetic patients than the diabetic group, the clinical outcome of the surgery was the same in both groups. Therefore, our results suggest that diabetic patients should be considered for CTR just as non-diabetic groups. For better results, we recommend that CTR should be applied as soon as possible (within three years after the initiation of symptoms), especially in patients who do not respond well to nonoperative treatments and their symptoms progress.

\section{Acknowledgements}

The authors express their thanks to all the medical personnel of the Orthopedic Surgery Ward in Chamran and Imam Reza hospitals for their cooperation. Special thanks to Dr. M. Jazayeri, professor of physiatry at Shiraz University of Medical Sciences for his cooperation at carrying out the electrodiagnostic evaluations of patients.

\section{Authors' Contributions}

Study concept and design: Kamran Mozaffarian, Mohammad Owjimehr, Behdad Eskandari Sani. Drafting of the manuscript: Mohammad Owjimehr, Behdad Eskandari Sani, Farzam Mokarami. Critical revision of the manuscript for important intellectual content: Kamran Mozaffarian, Mohammad Owjimehr, Behdad Eskandari Sani, Farzam Mokarami. Study supervision: Kamran Mozaffarian, Reza Sharifzadeh.

\section{References}

1. Canale ST, Beaty JH. Campbell's Operative Orthopaedics. 11th edPhiladelphia: Mosby; 2008.

2. Stanring S, Ellis H, Healy J, Johnson D. Gray's Anatomy. 39th edPhiladelphia: Churchill Livingstone; 2005.
3. Green DP, Hotchkiss RN, Pederson WC, Wolfe SW. Green's Operative Hand Surgery. 5th edPhiladelphia: Churchill Livingstone; 2005.

4. Thomsen NO, Englund E, Thrainsdottir S, Rosen I, Dahlin LB. Intraepidermal nerve fibre density at wrist level in diabetic and non-diabetic patients. Diabet Med. 2009;26(11):1120-6.

5. Thomsen NO, Rosen I, Dahlin LB. Neurophysiologic recovery after carpal tunnel release in diabetic patients. Clin Neurophysiol. 2010;121(9):1569-73.

6. Ozkul Y, Sabuncu T, Kocabey Y, Nazligul Y. Outcomes of carpal tunnel release in diabetic and non-diabetic patients. Acta Neurol Scand. 2002;106(3):168-72.

7. Mondelli M, Padua L, Reale F, Signorini AM, Romano C. Outcome of surgical release among diabetics with carpal tunnel syndrome. Arch Phys Med Rehabil. 2004;85(1):7-13.

8. Katz JN, Losina E, Amick BC, Fossel AH, Bessette L, Keller RB. Predictors of outcomes of carpal tunnel release. Arthritis Rheum. 2001;44(5):1184-93.

9. Thomsen NO, Cederlund R, Rosen I, Bjork J, Dahlin LB. Clinical outcomes of surgical release among diabetic patients with carpal tunnel syndrome: prospective follow-up with matched controls. J Hand Surg Am. 2009;34(7):1177-87.

10. Thatte MR, Mansukhani KA. Compressive neuropathy in the upper limb. Indian J Plast Surg. 2011;44(2):283-97.

11. Badger SA, O'Donnell ME, Sherigar JM, Connolly P, Spence RA. Open carpal tunnel release--still a safe and effective operation. Ulster Med J. 2008;77(1):22-4.

12. Bulut T, Sener U, Yagdi S, Kazimoglu C, Sener M. Relationship between clinical and electrophysiological results in surgically treated carpal tunnel syndrome. Eklem Hastalik Cerrahisi. 2011;22(3):140-4.

13. Bodavula VK, Burke FD, Dubin NH, Bradley MJ, Wilgis EF. A prospective, longitudinal outcome study of patients with carpal tunnel surgery and the relationship of body mass index. Hand (NY). 2007;2(1):27-33.

14. Yeo KQ, Yeo EM. Comparison of the results of open carpal tunnel release and KnifeLight carpal tunnel release. Singapore Med J. 2007;48(12):1131-5.

15. Wong KC, Hung LK, Ho PC, Wong JM. Carpal tunnel release. A prospective, randomised study of endoscopic versus limited-open methods. J Bone Joint Surg Br. 2003;85(6):863-8.

16. Gerritsen AA, Scholten RJ, Assendelft WJ, Kuiper H, de Vet HC, Bouter LM. Splinting or surgery for carpal tunnel syndrome? Design of a randomized controlled trial [ISRCTN18853827]. BMC Neurol. 2001;1:8.

17. Isaac SM, Okoro T, Danial I, Wildin C. Does wrist immobilization following open carpal tunnel release improve functional outcome? A literature review. Curr Rev Musculoskelet Med. 2010;3(14):11-7.

18. Huemer GM, Koller M, Pachinger T, Dunst KM, Schwarz B, Hintringer T. Postoperative splinting after open carpal tunnel release does not improve functional and neurological outcome. Muscle Nerve. 2007;36(4):528-31.

19. Kasper D, Braunwald E, Fauci A, Hauser S, Longo D. Harrison `s principles of internal medicine. 16th edNew York : McGraw Hill; 2005.

20. Mondelli M, Rossi S, Monti E, Aprile I, Caliandro P, Pazzaglia C, et al. Long term follow-up of carpal tunnel syndrome during pregnancy: a cohort study and review of the literature. Electromyogr Clin Neurophysiol. 2007;47(6):259-71. 